\title{
DESEMPENHO DOS DIFERENTES MÉTODOS DE AMOSTRAGEM PARA GARACTERIZAĢÃO DA ICTIOFAUNA ASSOCIADA AO MANGUEZAL DA PRAIA DE ARPOEIRAS EM ACARAÚ, CEARÁ
}

\author{
Performance of the different sampling methods in the \\ characterization of ictiofauna associated with the mangrove \\ in the Arpoeiras beach, Acaraú, Ceará \\ José Ivan Fonteles de Vasconcelos Filho ${ }^{1 *}$, Rafaela Camargo Maia², Rodrigo Salles ${ }^{3}$ \\ ${ }^{1}$ Mestrando em Engenharia de Pesca pela Universidade Federal do Ceará, Bolsista CNPq, Universidade Federal do \\ Ceará, Endereço: Av. Mister Hull, s/n - Pici - CEP 60455-760 - Fortaleza - CE, ivanfontelesbio@gmail.com \\ ${ }^{2}$ Professora do Instituto Federal de Educação, Ciência e Tecnologia do Ceará - Campus Acaraú, \\ Instituto Federal de Educação, Ciência e Tecnologia do Ceará - Campus Acaraú, \\ Av. Des. Armando de Souza Louzada - Sítio Buriti, Acaraú - CE, 62580-000, rafaelacmaia@yahoo.com.br \\ ${ }^{3}$ Professor do Instituto Federal de Educação, Ciência e Tecnologia do Ceará - Campus Paracuru, \\ Instituto Federal de Educação, Ciência e Tecnologia do Ceará - Campus Paracuru, \\ Rua Dez, Paracuru - CE, 62680-000, rdesalles@hotmail.com
}

\begin{abstract}
RESUMO
Os manguezais são importantes não apenas devido à sua biodiversidade, mas também por sua importância econômica, uma vez que as comunidades que vivem em seu entorno dependem direta e indiretamente da exploração de seus recursos naturais, entre eles os peixes. Nesse contexto, o objetivo do presente trabalho foi caracterizar a ictiofauna do manguezal da praia de Arpoeiras, em Acaraú, CE, bem como testar os métodos mais adequados para amostragem nesse ambiente. Foram utilizadas diferentes artes de pesca, como anzóis, tarrafas e armadilhas (manzuá). Um total de 256 indivíduos foram capturados durante o período de amostragem, distribuídos em 12 espécies, 10 famílias, 5 ordens e 1 classe, com alta dominância para as espécies Lutjanus alexandrei $(24,6 \%)$, Lutjanus jocu $(24,2 \%)$, Eucinostomus argenteus $(25,7 \%)$ e Diapterus auratus $(8,9 \%)$. A tarrafa foi o equipamento de pesca mais eficiente devido à sua baixa seletividade em comparação com outras artes de pesca utilizadas. Análises referentes ao estágio de maturação, peso $(\mathrm{g})$ e tamanho $(\mathrm{cm})$ dos espécimes coletados mostraram que todos os animais eram sexualmente imaturos. Esses
\end{abstract}

Recebido em: 15/02/2019

Aprovado em: 05/06/2019

Publicado online em: $1^{\circ} . / 11 / 2019$ 
dados confirmam que manguezais são áreas de berçários para peixes, inclusive para espécies de interesse econômico, sendo necessárias medidas para sua conservação.

Palavras-chave: caracterização, ictiofauna, manguezal.

\begin{abstract}
Mangroves are important, not only because of their biodiversity, but also because of their economic importance, to the communities that live in their surroundings which depend directly and indirectly on the exploitation of their natural resources, e.g., fishes. Thus, this study aimed to characterize the ichthyofauna of the mangrove of Arpoeiras beach, in Acaraú - CE, and, to test the most suitable methods for sampling in this environment. Differents fishing gear were used as hooks, casting net and trap (manzuá). A total of 256 individuals were caught during the sampling period, distributed in 12 species, 10 families, 5 orders and 1 class, with high dominance for the species Lutjanus alexandrei (24.6\%); Lutjanus jocu (24.2\%); Eucinostomus argenteus (25.7\%) and Diapterus auratus $(8.9 \%)$. The casting net was the most efficient fishing gear due to its low selectivity in comparison with others fishing gear used. Analysis of the maturation stage, weight $(\mathrm{g})$ and size $(\mathrm{cm})$ of the specimens collected showed that all animals were sexually immature. These data confirm that mangroves are nursery areas for fish, including for species of economic interest, and conservation measures are necessary.
\end{abstract}

Keywords: characterization, ichthyofauna, mangrove.

\title{
INTRODUÇÃO
}

O manguezal é um ambiente de transição entre as zonas costeira e marinha, característico de regiões tropicais (Tomlinson, 2016), sendo caracterizado por uma vegetação lenhosa típica, associada a outros componentes da flora e fauna, adaptado às condições limitantes de salinidade, substrato inconsolidado, pouco oxigenado e com frequente submersão pelas marés (Duke, 2017). Ocupa a segunda posição em termos de produtividade bruta, superado somente pelos recifes de corais, sendo considerado como áreas de berçários para muitas espécies animais (Sheridan \& Hays, 2003).

Esses ecossistemas fornecem uma grande quantidade de bens e serviços ambientais, como proteção costeira, purificação de água, sequestro de carbono, pesca, matéria-prima e atividades relacionadas ao turismo, influenciando direta e indiretamente o bem-estar humano e a regulação de serviços em áreas adjacentes (Brehmer et al., 2013; Lee et al., 2014). Entretanto, sua biodiversidade está sendo alterada pela ação antropogênica do desmatamento, pesca predatória e principalmente pela falta de manejo sustentável (Rezende et al., 2015).

Entre as diversas razões para se estudar os manguezais, destaca-se o fato de que muitas comunidades humanas têm uma dependência tradicional desses ecossistemas para sua subsistência (Alves \& Nishida, 2001). Cerca de 95\% da produção de alimento marinho está relacionada ao manguezal (Souza et al., 2018). Logo, é possível afirmar que há uma ligação direta entre os bosques de mangue e as pescarias costeiras, destacando a importância dos manguezais como fonte de manutenção dos recursos pesqueiros explorados pelas comunidades de pescadores locais (Manson et al., 2005; Santo \& Lana, 2017; Tran \& Fischer, 2017). No entanto, os aspectos ecológicos dessa ligação ainda são mal compreendidos, ficando limitado a poucas informações sobre espécies de peixes de valor comercial. 
Estudos realizados por Benzeev, Hutchinson e Friess (2017) investigaram o impacto do desenvolvimento urbano na composição da comunidade de peixes e tamanho dos indivíduos em manguezais adjacentes e locais de costa rochosa artificial em Singapura-Ásia; Chaves e Serenato (1998) analisaram a diversidade de dietas na assembleia de linguados no manguezal da Baía de Guaratuba-PR; Neves et al. (2006) investigaram o uso do manguezal de Guaratiba, na Baía de Sebetiba-RJ, pelo peixe rei (Atherinella brasilienses); Chaves e Vendel (2008) realizaram análise comparativa da alimentação de peixes entre ambientes de marisma e de manguezal no estuário da Baía de Guaratuba-PR e Osório; Godinho e Lotufo (2011) caracterizaram a ictiofauna associada às raízes de mangue no estuário do rio Pacoti-CE.

O estudo da diversidade de peixes e seus padrões de variações espacial e temporal são de extrema relevância para a análise da qualidade ambiental, possibilitando o desenvolvimento de planos que minimizem a degradação e regulamentem a utilização dos recursos hídricos (Teixeira et al., 2005). Contudo, há uma carência de informações sobre os procedimentos metodológicos para ambientes de manguezal.

Experimentos realizados por Cattani et al. (2012), Duarte, Deus e Py-Daniel (2012) e Portella e Medeiros (2015), comparando a eficiência de diversas artes de pesca na captura de espécimes de pescado, constataram que utilizar diferentes petrechos de coleta pode diminuir a seletividade inerente de cada um desses aparelhos, melhorando a efetividade de amostragens em ambientes pouco estudados. Dessa forma, determinar métodos de coleta que sejam específicos para a comunidade íctia nessas áreas é necessário devido à escassez de informações em relação a esse tema e de sua significativa relevância ecológica.

Assim, neste trabalho foram testadas as seguintes hipóteses científicas: (i) a ictiofauna associada ao manguezal de Arpoeiras é composta por peixes jovens que, quando adultos, habitam os ambientes marinho e límnico associados; (ii) a riqueza e a abundância da ictiofauna estimada no manguezal dependem dos diferentes métodos de amostragem utilizados; e (iii) existem espécies de peixes endêmicas do manguezal que habitam esse ecossistema ao longo de todo o seu ciclo de vida.

Nesse contexto, o objetivo do presente trabalho foi caracterizar a ictiofauna do manguezal da praia de Arpoeiras, no município de Acaraú-CE, bem como testar os métodos mais adequados para a amostragem de peixes em manguezais. Especificamente, pretendeu-se: (i) identificar taxonomicamente a ictiofauna associada à área de manguezal estudada; (ii) avaliar a abundância e riqueza de peixes coletados no manguezal nos diferentes métodos de amostragem utilizados; e (iii) avaliar o tamanho, o peso, a maturidade sexual e a proporção sexual dos indivíduos coletados ao longo do período amostral.

\section{MATERIAL E MÉTODOS}

\section{Área de estudo}

O município de Acaraú, no litoral oeste do estado do Ceará, está a aproximadamente 260 quilômetros de Fortaleza, capital do estado, sendo banhado pelo mar e pelo rio Acaraú, o segundo maior rio do estado. Apresenta uma temperatura média de $28^{\circ} \mathrm{C}$ e pluviosidade média de 125,9 mm anuais (Funceme, 2017). Sua população em 2018 é de 62.557 habitantes (IBGE, 2010) e sua economia é baseada, principalmente, na carcinicultura e na atividade pesqueira artesanal (Araújo et al., 2007; Saraiva, 2015). 
O desmatamento dos manguezais é um dos principais problemas quanto à degradação e à descaracterização da paisagem na região, principalmente em função da implantação de empreendimentos de carcinicultura (Maia, 2016; Thiers; Meireles \& Santos, 2016).

As amostragens foram realizadas em um canal de maré localizado no manguezal do estuário do rio Acaraú (S 2050'17.458”, W 400456.470”), próximo à praia de Arpoeiras. O canal estudado possui extensão de 200 metros e situa-se dentro de um bosque de mangue com uma densa vegetação, dominada por Rhizophora mangle, tornando essa área bem protegida e com pouca influência antrópica.

\section{Metodologia}

As coletas foram realizadas mensalmente no período de junho a novembro de 2017, em marés de sizígia diurnas.

A amostragem da ictiofauna foi realizada com quatro artes de pesca distintas (anzóis, tarrafa, manzuás e rede de arrasto) com o intuito de determinar quais os métodos mais adequados para a coleta de peixes em ambientes de manguezais. A escolha dos petrechos de pesca foi determinada após conversas informais com pescadores locais na área selecionada.

Anzóis - A pesca com anzol é um método simples e eficiente, porém bastante seletivo. Dessa forma, foram realizadas conversas informais com pescadores da região sobre os tamanhos de anzóis mais adequados para a utilização no experimento.

A pescaria foi realizada por quatro pessoas e de forma aleatória ao longo do canal, sendo todos os anzóis iscados com camarão. Foram usados dois anzóis $\mathrm{n}^{\mathrm{0}} 12$ e dois anzóis $\mathrm{n}^{\circ} 14$, com esforço amostral de 45 minutos.

Tarrafa - Durante as pescarias com tarrafa foram realizados 10 lances ao longo do canal, de forma aleatória e em locais com profundidade abaixo de 1,5 metros. Os arremessos que não abriram de forma adequada foram desconsiderados e repetidos. A tarrafa usada para a pescaria foi confeccionada com nylon 50 , possuía 2,5 metros de comprimento e três metros de diâmetro, com malhas de 15 milímetros (entre nós opostos).

Manzuás - Foram utilizados três manzuás com malhas de 20 milímetros (entre nós opostos). Cada armadilha foi fixada de forma aleatória em profundidades abaixo de 1,5 metros e iscada com camarão. As iscas foram colocadas dentro de sacos de nylon e amarradas no interior de cada armadilha. A despesca foi realizada 24 horas após a sua armação.

Rede de arrasto - A rede de arrasto utilizada possuía 4 metros de comprimento e 1,5 metros de altura, com malhas de $20 \mathrm{~mm}$ (entre nós opostos) e hastes de madeira em suas extremidades para facilitar seu manuseio e manter a rede aberta. $\mathrm{O}$ arrasto foi realizado de forma manual por duas pessoas. O esforço amostral foi de cinco minutos por arrasto e em profundidades abaixo de 1,5 metros. Foi realizado um total de quatro arrastos por coleta, sendo todos de forma aleatória.

Todos os peixes coletados foram colocados em sacos plásticos devidamente identificados e acondicionados em caixas de isopor com gelo para posterior análise.

\section{Procedimentos em laboratório}

Em laboratório, os peixes foram congelados para preservar suas características físicas. No dia posterior à coleta, os peixes foram descongelados e identificados ao nível de espécie 
de acordo com Szpilman (2000), Araújo, Texeira e Oliveira (2004), Moura e Lindeman (2007), Lessa e Nóbrega (2009), Meneses et al. (2015), Melo et al. (2015) e FishBase (2017).

Após a identificação, foram obtidos o comprimento padrão e o comprimento total de cada espécime, com o auxílio de um ictiômetro. Em seguida, foram pesados em uma balança de precisão $(0,001 \mathrm{~g})$. Os resultados obtidos foram registrados e organizados em tabelas para posterior análise estatística.

Todos os peixes capturados foram abertos para verificação visual do estágio de maturação sexual. As características utilizadas para definição macroscópica dos estádios de maturação gonadal se basearam na metodologia descrita por Vazzoler (1996). As gônadas foram retiradas da cavidade celomática, realizando uma incisão ventral a partir da abertura urogenital, em direção à cabeça com um bisturi cirúrgico, de forma cuidadosa para não danificá-las.

A seguir, os peixes foram fixados em formol (10\% de formaldeído).

\section{Análises estatísticas}

Quanto à estatística, primeiramente foram testadas a normalidade e a homogeneidade dos dados. A seguir, foi realizada uma análise descritiva da riqueza e abundância de peixes entre as artes de pesca testadas e os meses de amostragem.

Para comparar o tamanho (comprimento padrão) e o peso das espécies dominantes entre os meses de amostragem (junho a novembro) foi utilizada uma Análise de Variância (Anova). Quando detectadas diferenças entre as médias, ao nível de significância de 5\% $(\mathrm{p}<0,05)$, o teste de comparações múltiplas de Tukey foi utilizado.

Para subsidiar a seleção das variáveis de tamanho a serem usadas nessa análise, utilizou-se a uma correlação entre todas as variáveis, retirando das análises os parâmetros altamente correlacionados $(r>0,95)$. Assim, só foi utilizado o comprimento padrão. Como critério para determinar a dominância entre as espécies capturadas ao longo do trabalho, foram consideradas dominantes aquelas que ocorreram em $50 \%$ ou mais dos meses amostrados, resultando em quatro espécies: Lutjanus alexandrei, Lutjanus jocu, Eucinostomus argenteus e Diapterus auratus.

\section{RESULTADOS E DISCUSSÃO}

Foram capturados um total de 256 indivíduos, distribuídos em 12 espécies, 10 famílias, 5 ordens e 1 classe (Tabela I).

A maior riqueza de espécies foi da família Lutjanidae, com 2 espécies: Lutjanus jocu (carapitanga) e Lutjanus alexandrei (baúna-de-fogo); e da família Gerreidae, também com 2 espécies: Eucinostomus argenteus (carapitu) e Diapterus auratus (carapeba).

Essas espécies são comuns em ambientes costeiros como estuários e manguezais, principalmente na região Nordeste do Brasil. Estudos de caracterização da ictiofauna foram realizados por Araújo, Teixeira e Oliveira (2000) e Basilio et al. (2009) nos estuários do estado do Ceará; Xavier et al. (2012), nos manguezais de Mamanguape e por Grande et al. (2018) em Tamandaré, ambos no Nordeste do Brasil, registraram esses organismos entre os mais abundantes. 
Tabela I - Listagem em ordem decrescente de abundância da ictiofauna coletada no manguezal da Praia de Arpoeiras, no município de Acaraú - CE

\section{FILO CHORDATA}

Classe Actinopiterygii

$$
\text { Ordem Perciforme }
$$

Família Lutjanidae

Família Gerreidae

Litjanus jocu (Bloch e Schneider, 1801).

Lutjanus alexandrei Moura e Lindeman, 2007.

Família Gerreidae
Diapterus auratus Ranzani, 1840.
Eucinostomus argenteus Baird e Girard, 1855.

Família Centropomidae

Centropomus parallelus Poey, 1860.

Família Carangidae

Caranx latus Agassiz, 1831.

Família Sphyraenidae

Sphyraena barracuda (Walbaum, 1792).

Família Haemulidae

Ordem Clupeiforme

$$
\text { Haemulon parra (Desmarest, 1823) }
$$

Família Engraulidae

Ordem Mugiliforme Anchoviella lepidentostole (Fowler, 1911).

Ordem Beloniforme

Família Mugilidae

Mugil curema Valenciennes, 1836.

Família Belonidae

Ordem Tetraodontiforme

Strongylura marina (Walbaum, 1792).

Família Tetraodontidae

Sphoeroides testudineus (Linnaeus, 1758)

Em relação à representação de cada espécie, considerando o número total de peixes capturados (Figura 1), foram obtidos os seguintes resultados: Lutjanus alexandrei com 63 indivíduos, Lutjanus jocu: 62, Eucinostomus argenteus: 66 e Diapterus auratus com 23 espécimes.

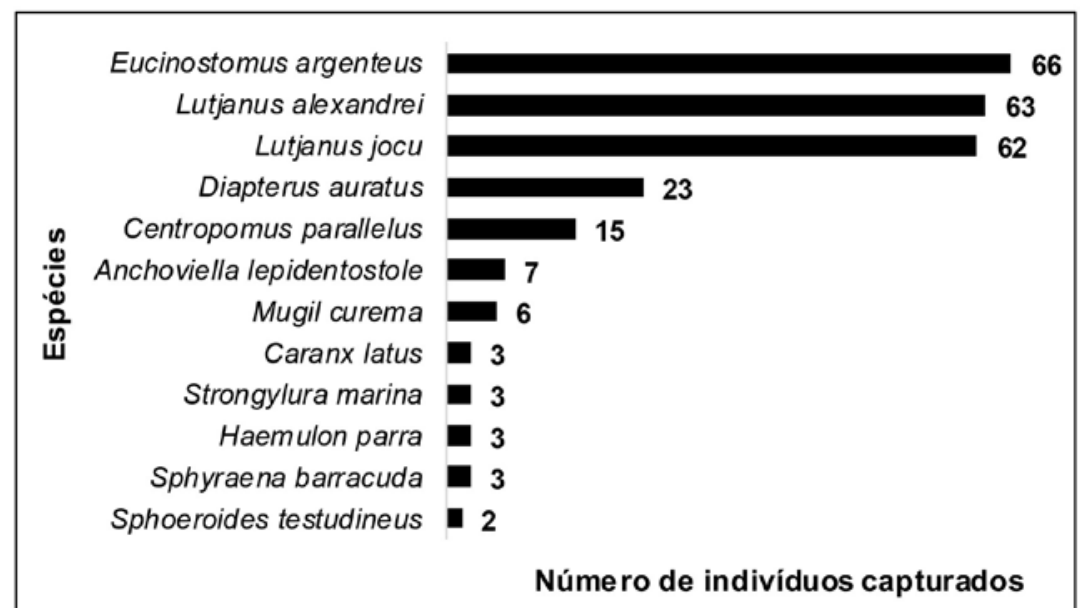

Figura 1 - Ranking das espécies de peixes capturadas durante as coletas no manguezal da Praia de Arpoeiras, no município de Acaraú - CE

A maior abundância do gênero Lutjanus nos manguezais do Nordeste brasileiro, tal qual registrados neste estudo, não é algo isolado, sendo considerado um padrão ecológico dessas espécies (Osório; Godinho \& Lotufo, 2011). Os peixes vermelhos, como são conhe- 
cidos os membros dessa família (Lutjanidae), são considerados espécies-chave e predadores de topo nos ambientes costeiros, constituindo importantes recursos pesqueiros nas regiões tropicais e subtropicais (Moraes, 2012). Durante estudos de caracterização da ictiofauna realizados por Lira e Teixeira (2008) na Praia de Jaguaribe, PE, Oliveira-Silva, PesoAguiar e Lopes (2008) no estado da Bahia, Moraes et al. (2009) na Praia de Ilhéus, BA, e por Pinheiro e Farias (2016) no Porto do Mucuripe, Fortaleza-CE, também foram registrados esse gênero entre os mais abundantes.

Por sua vez, a família Gerreidae, representada neste trabalho por D.auratus e E. argenteus, foi a segunda mais abundante durante todo período de coleta. Esses peixes podem ser encontrados ao longo de toda a costa brasileira, sendo amplamente distribuídos e apresentando altas abundâncias nesses ecossistemas (Paiva; Chaves \& Araújo, 2008), em parte, devido à sua capacidade de suportar variações adversas de salinidade, resultado de adaptações fisiológicas em resposta à salinidade do ambiente (Pereira \& Pessanha, 2017). Paiva, Chaves e Araújo (2008), ao avaliar a diversidade de peixes no estuário do Rio Formoso - PE, Ignácio e Spach (2009), no estuário da Baia de Paranaguá - PR, e Mattos et al. (2012), no manguezal da Reserva Ponta do Tubarão - RN, reforçaram a abundância dessa família nesses ambientes.

Estudos realizados com currais de pesca na comunidade de Ilha dos Coqueiros, também em Acaraú-CE, por Masih-Neto et al. (2017), a aproximadamente dois quilômetros da referida área de coleta, constataram a ocorrência e captura de todas as espécies amostradas na área de manguezal estudada, tendo as familias Lutjanidae e Gerreidae, novamente, como as mais abundantes.

Entre as artes de pesca experimentadas, todas, com exceção da rede de arrasto, mostraram-se eficientes no processo de captura da ictiofauna em ambientes de manguezal. Foi realizada apenas uma coleta com essa arte de pesca, uma vez que logo após a primeira coleta a rede estava danificada demais para ser consertada, optando-se por não a utilizar outra vez, uma vez comprovada sua ineficiência para este ambiente. Isso devido à grande quantidade de matéria orgânica e outros materiais em suspensão. $\mathrm{O}$ arrasto torna a rede pesada e lenta, impossibilitando uma velocidade adequada para a captura dos peixes no ambiente amostrado. As malhas da rede foram facilmente rasgadas pela bioincrustação de ostras e cracas presente nas raízes do bosque de mangue.

Os anzóis $\mathrm{n}^{\mathrm{O}} 12$ amostraram três espécies de peixes associadas ao manguezal, enquanto os anzóis $n^{\circ} 14$ capturaram apenas duas espécies (Figura 2). No entanto, o anzol no 14 mostrou maior abundância em relação ao número de indivíduos capturados. A porcentagem do número de indivíduos capturados para o anzol $\mathrm{n}^{\mathrm{o}} 12$ foi de 4,29\% do total $(\mathrm{N}=$ $11)$, enquanto para o anzol de $\mathrm{n}^{\mathrm{o}} 14$ foi de $6,25 \%$ do total $(\mathrm{N}=16)$.

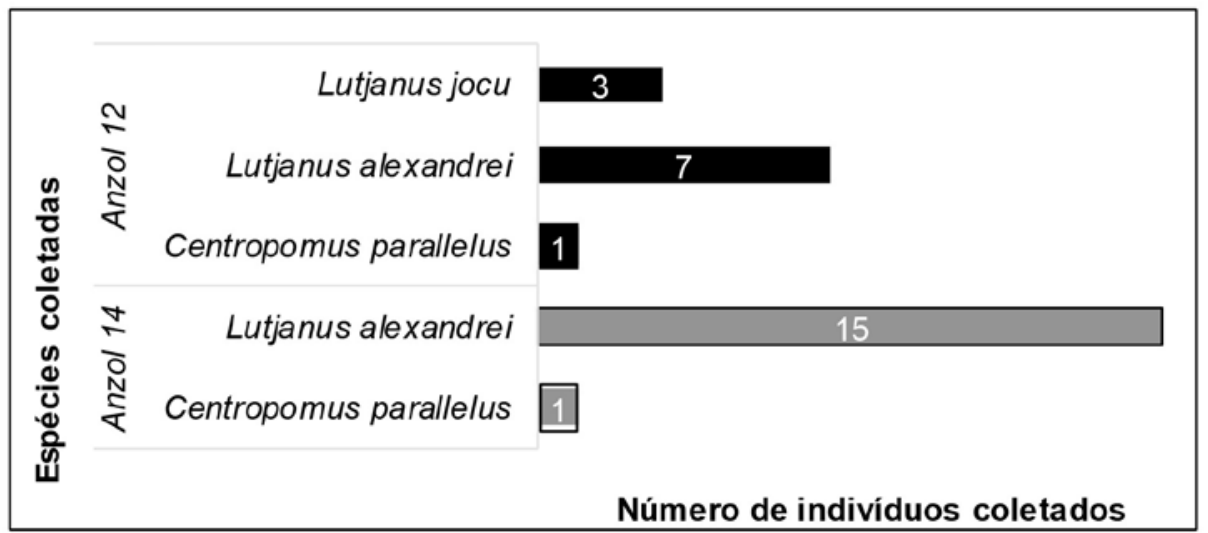

Figura 2 - Riqueza e abundância da ictiofauna capturada com anzóis no manguezal da praia de Arpoeiras, no município de Acaraú - CE. 
Os aparelhos que não retêm organismos em seu interior, como os anzóis, são mais seletivos (Fonteles-Filho, 2011). Isso ocorre devido ao fato de os peixes pequenos não conseguirem morder o conjunto anzol/isca; em contrapartida, os peixes grandes, por terem uma boca maior, dificilmente são capturados por anzóis pequenos (Hamley, 1975). Desse modo, por se tratar de um ambiente de manguezal, onde a maior parte de sua ictiofauna é composta por organismos jovens (Sheridan \& Hays, 2003), é possível deduzir que a relação entre o tamanho da boca e o tamanho dos anzóis utilizados tenha conferido ao anzol de menor porte $\left(\mathrm{n}^{\mathrm{o}} 14\right)$ maior eficiência em relação ao anzol $\mathrm{n}^{\mathrm{o}} 12$.

Um outro fator que pode ter sido responsável pela baixa riqueza de indivíduos capturados pelos anzóis deve-se ao uso de apenas um tipo de isca durante as pescarias (camarão). A utilização de uma maior variedade de iscas poderia aumentar consideravelmente a diversidade de organismos, assim como o número de organismos capturados (Catella, 2007).

Por sua vez, os manzuás (armadilhas do tipo covo) amostraram duas espécies de peixes da família Lutjanidae, $L$. jocu $(\mathrm{N}=31)$ e L. alexandrei $(\mathrm{N}=33)$, registrando abundância de 64 indivíduos, equivalendo a 25\% do número total de organismos coletados.

A baixa riqueza e abundância de peixes obtidos pelos manzuás, a exemplo dos anzóis, também pode ser justificada pelo fato de ter sido utilizada apenas um tipo de isca, promovendo a seletividade dos organismos de acordo com seus hábitos alimentares. Em estudo realizado por Possamai, Rosa e Corrêa (2014) na Gamboa do Perequê-PR, foram utilizados quatro tipos de atrativos (fígado bovino cru, quirera grossa, ração para cães sabor carne e massinha para pesca de lambari), a fim de testar a capacidade de atração de cada isca. Nesse experimento, verificou-se que nenhum dos quatro atrativos se destacou quanto à riqueza e abundância de indivíduos capturados. Contudo, cada um deles mostrou-se eficiente na atração de grupos específicos de organismos. Assim, a eficiência de captura de cada armadilha dependerá principalmente do tipo de atrativo utilizado, o qual deverá corresponder às características alimentares das espécies-alvo, de modo que uma maior variabilidade de iscas provavelmente ampliaria o número de espécies capturadas (Sanches \& Sebastiani, 2009; Rocha, 2010).

Outro fator que contribuiu para a obtenção desses resultados são as taxas de escape dessas armadilhas. Em uma avaliação realizada por Ribeiro e Zuanon (2006), averiguou-se a frequência de escapes de armadilhas do tipo covo. $\mathrm{O}$ experimento foi realizado durante o período matutino e durou cinco horas. A cada hora todas as armadilhas eram revistadas, anotando-se as espécies e o número de indivíduos capturados. A taxa de escape foi medida pela diferença de captura em cada armadilha em horários subsequentes. Nas 40 vistorias sucessivas realizadas detectou-se a ocorrência de escapes em 11 ocasiões (27\%), representando uma perda potencial de 21 peixes. Desse modo, o período de 24 horas, embora confira maior tempo de atuação aos aparelhos de pesca, também aumenta a possibilidade de fuga dos indivíduos.

No que diz respeito à tarrafa, houve riqueza de 12 espécies e abundância de 151 indivíduos capturados (Figura 3). Sua porcentagem referente ao número total de indivíduos durante todos os meses de coleta foi de $58,98 \%$.

Os indivíduos se tornam mais vulneráveis quanto menos selecionados forem pelo aparelho de pesca utilizado (Fonteles-Filho, 2011). Dessa forma, a tarrafa foi a arte de pesca mais eficiente em relação às demais utilizadas. $O$ tamanho reduzido de sua malha permitiu a captura de forma não seletiva de todo o recurso pesqueiro cercado pela rede (Giglio \& Freitas, 2013). Em pescarias realizadas, Torres et al. (2007) na praia do muni- 
cípio de Porto de Pedras, em Alagoas, e Duarte, Deus e Py-Daniel (2012) nas praias no baixo rio Purus, no Amazonas, avaliaram a eficiência de petrechos de pesca diferentes na captura de diversas espécies de peixes, verificando que quanto menor a malha das tarrafas, menor a seletividade do aparelho. Outro fator que conferiu a tarrafa maior eficiência foi o fato de esta, entre todas as artes de pesca testadas, ser a única com método ativo de amostragem. A diferença significativa tanto para riqueza quanto para abundância registrada nesse trabalho indica que os métodos passivos foram menos eficientes que o método ativo.

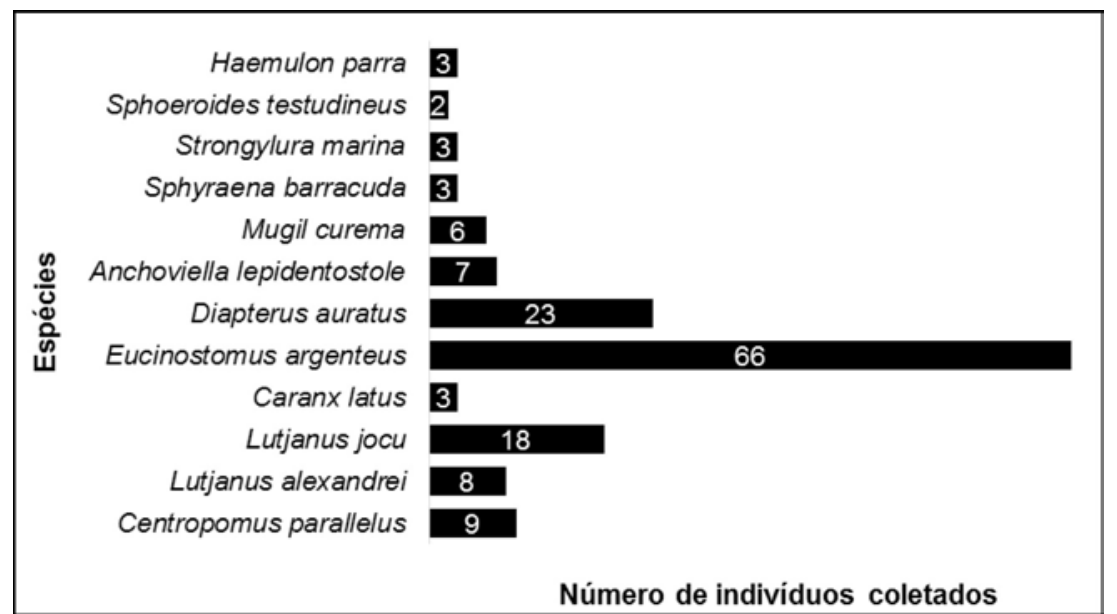

Figura 3 - Riqueza e abundância da ictiofauna capturada com a tarrafa no manguezal da Praia de Arpoeiras, no município de Acaraú - CE

É possível que as próprias características do ambiente tenham afetado o rendimento dos petrechos com métodos passivos, não apenas pela complexidade estrutural do bosque de mangue, mas principalmente devido às altas taxas de alimento disponíveis no local, tornando as iscas usadas nos anzóis e manzuás menos atrativas. Além disso, os peixes também são atraídos pelo abrigo e sombra que as armadilhas proporcionam (Gomes et al., 1998), contudo, isso se torna irrisório nos manguezais, quando comparados a outros hábitats, devido à abundância natural dessas condições. Entretanto, o uso conjunto dos diferentes métodos (ativos e passivos), tal qual realizado nesta pesquisa, diminui a possibilidade de vícios amostrais e torna-se complementar ao final das coletas. Consequentemente, é importante levar em consideração o tipo de aparelho utilizado em cada amostragem, pois cada um é utilizado para amostrar uma parcela específica da população (Vazzoler, 1996; Ribeiro; Zuanon, 2006; Pichler et al., 2017).

Entre os meses amostrados, julho foi o que apresentou maior riqueza de espécies ( $\mathrm{N}$ $=7$ ) e o mês de junho o que apresentou menor riqueza $(\mathrm{N}=3)$ (Figura 4). O mês de novembro mostrou-se com a maior abundância $(\mathrm{N}=69)$ e a menor abundância foi registrada no mês de outubro $(\mathrm{N}=33$ ) (Figura 4 ).

Algumas comunidades de peixes apresentam uma tendência de se movimentar em direção aos manguezais durante o período seco para desovar e buscar abrigo em sua fase larval e, durante o período chuvoso, com a forte redução da salinidade, os peixes se movimentam para as áreas mais costeiras (Barletta et al., 2005). No entanto, não foi possível determinar nenhum padrão de distribuição entre as espécies amostradas, senão a dominância do gênero Lutjanus em todos os meses de amostragem e sobre todas as artes de pesca utilizadas. 


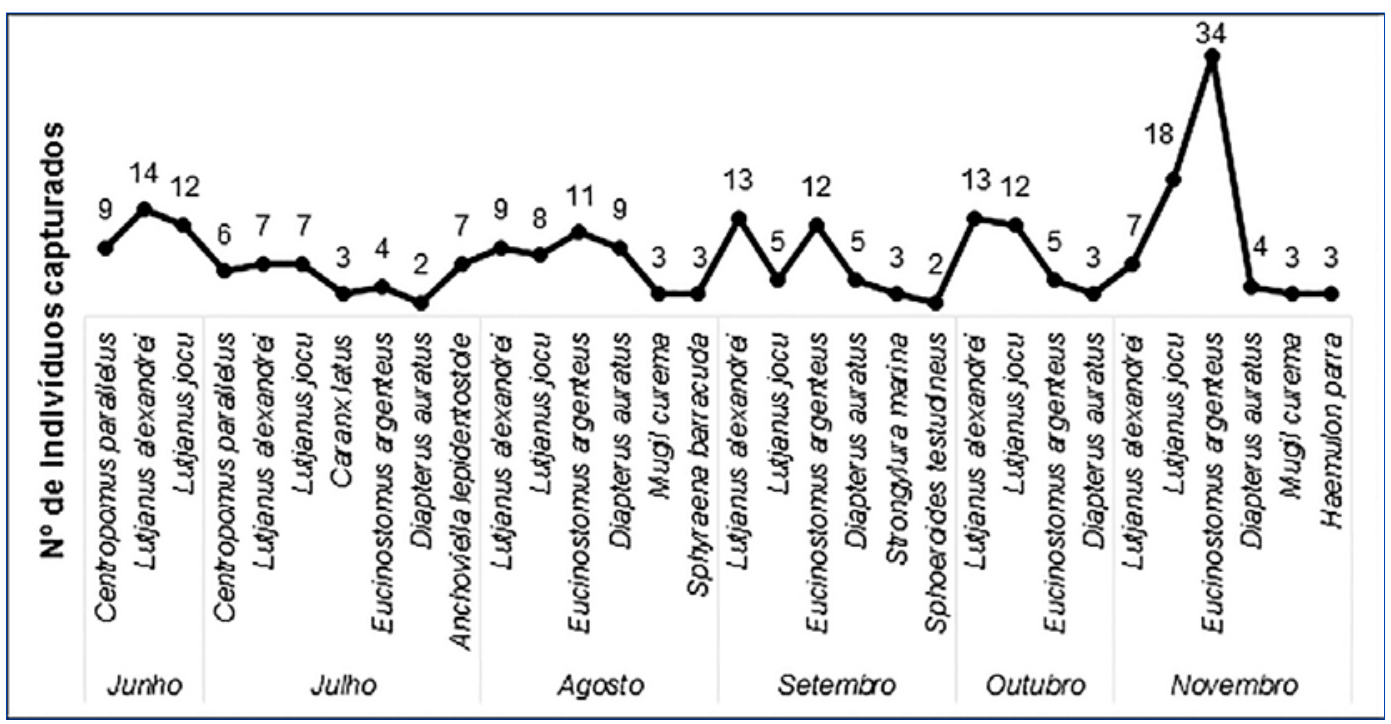

Figura 4 - Riqueza e abundância da ictiofauna capturada nos meses de junho a novembro nas artes de pesca no manguezal da Praia de Arpoeiras, Acaraú - CE

Com relação ao tamanho, entre os organismos amostrados, a espécie L. alexandrei apresentou o maior desvio padrão em relação às demais espécies, indicando maiores índices de desuniformidade de tamanhos dentro da classe de comprimento dessa espécie. Enquanto, D. auratus e E. argenteus apresentaram pouca variação de tamanho ao longo de todo período de coletas, exibindo os menores desvios padrões (Tabela II).

\begin{tabular}{|c|c|c|}
\hline Espécie & Comprimento médio & Desvio padrão \\
\hline L. alexandrei & $10,33 \mathrm{~cm}$ & $\pm 35,89$ \\
\hline L. jocu & $11 \mathrm{~cm}$ & $\pm 2,61$ \\
\hline D. auratus & $6 \mathrm{~cm}$ & $\pm 0,96$ \\
\hline E. argenteus & $7,42 \mathrm{~cm}$ & $\pm 0,97$ \\
\hline
\end{tabular}

Os resultados indicaram diferenças significativas para o comprimento padrão ao longo do período amostral em duas espécies: D. auratus $\left(\mathrm{F}_{4,18}=5,3978, \mathrm{p}=0,0490\right.$ (Figura 5) e L. jocu $\left(\mathrm{F}_{5,56}=4,3555, \mathrm{p}=0,0203\right)$ (Figura 6). O mês de agosto apresentou maiores valores de tamanho para a espécie D. auratus, seguido pelo mês de outubro. Os outros meses mostraram-se estatisticamente semelhantes. O mês de agosto também apresentou os indivíduos de maior tamanho para L. jocu, seguido pelo mês de outubro. Os meses de junho, julho e setembro foram estatisticamente semelhantes e o mês de novembro apresentou os organismos de menor tamanho para essa espécie. Para as demais espécies não foram observadas diferenças significativas em relação ao seu tamanho durante o presente estudo; E. argenteus $\left(\mathrm{F}_{4,61}=0,21023, \mathrm{p}=0,93180\right)$ e L. alexandrei $\left(\mathrm{F}_{5,57}=1,4263, \mathrm{p}=0,22872\right)$.

Dentre os organismos dominantes, o maior desvio padrão em relação ao peso dos elementos coletados foi o do L. jocu, que apresentou a maior variação ao longo do período amostral. Enquanto os membros da família Gerreidae: E. argenteus e D. auratus apresentaram os menores desvios padrões, devido a uma maior uniformidade dos pesos dos indivíduos coletados (Tabela III). 


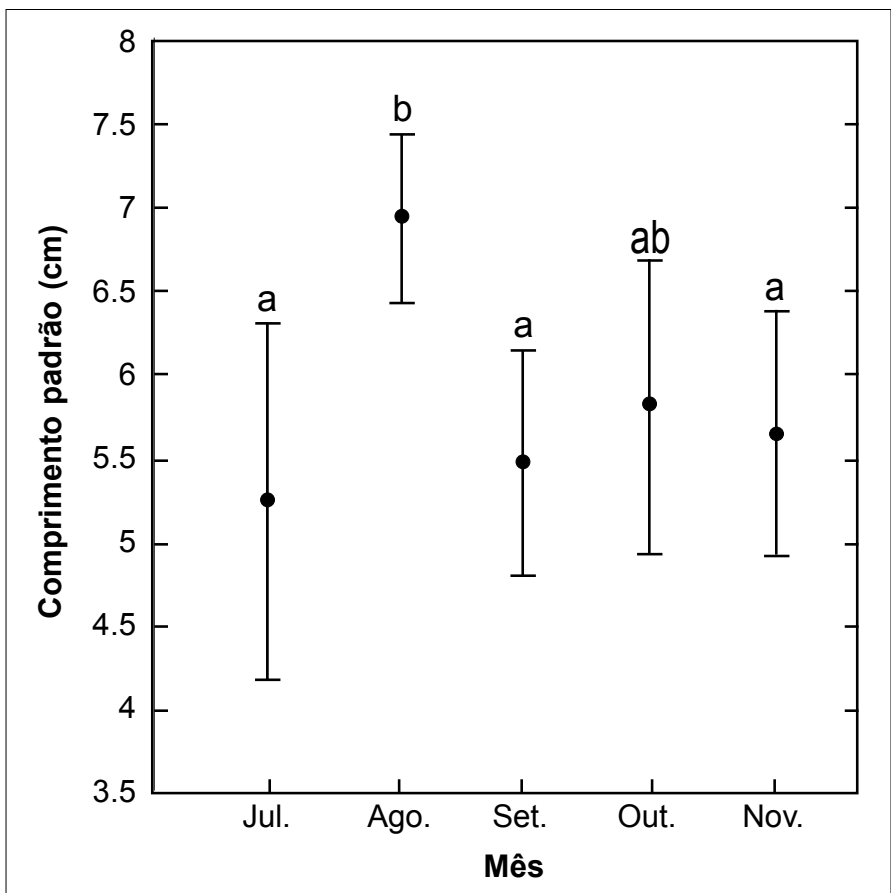

Figura 5 - Comprimento padrão \pm erro padrão (cm) de D. auratus ao longo dos meses amostrais (junho a novembro) no manguezal da Praia de Arpoeiras, no município de Acaraú - CE Nota: letras diferentes indicam diferenças significativas de acordo com o teste Tukey de comparações múltiplas.

Figura 6 - Comprimento padrão + erro padrão $(\mathrm{cm})$ de $L$. jocu ao longo dos meses amostrais (junho a novembro) no manguezal da praia de Arpoeiras, no município de Acaraú - CE

Nota: letras diferentes indicam diferenças significativas de acordo com o teste Tukey de comparações múltiplas.

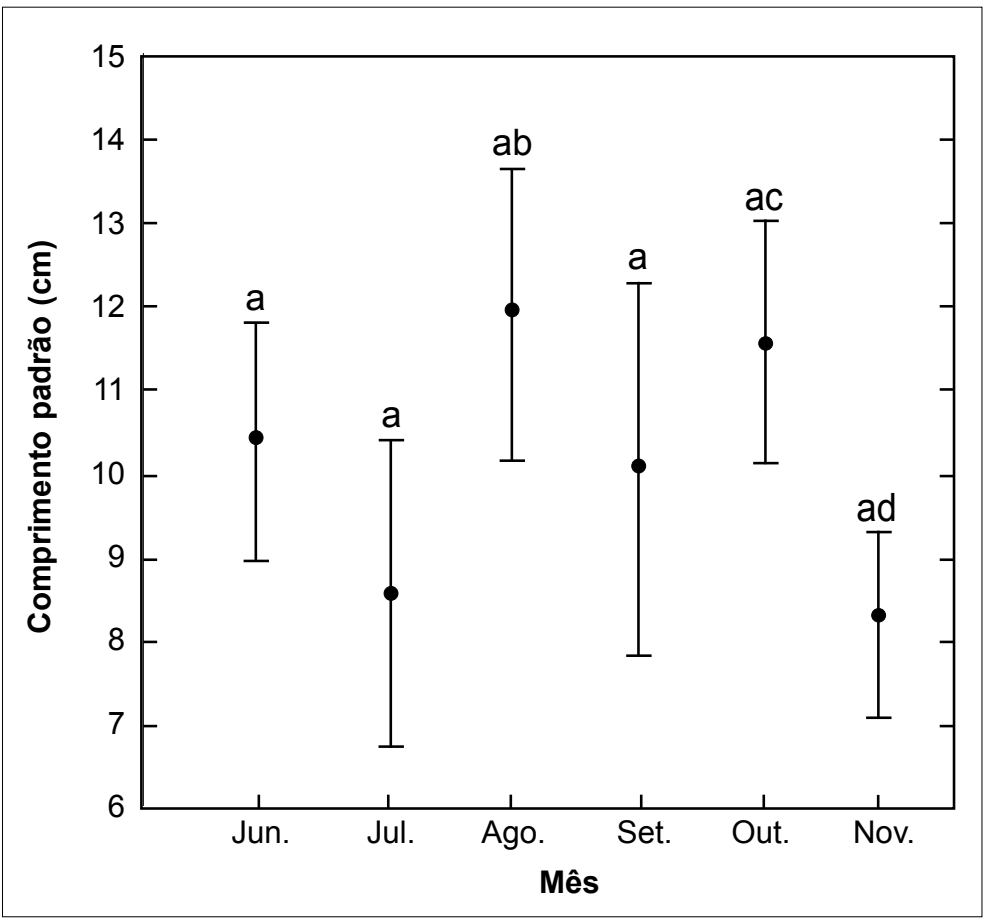

Tabela III - Peso médio e desvio padrão das espécies dominantes coletadas no manguezal da Praia de Arpoeiras, no município de Acaraú - CE

\begin{tabular}{ccc}
\hline Espécie & Peso médio & Desvio padrão \\
L. alexandrei & $42,35 \mathrm{~g}$ & $\pm 24,74$ \\
L. jocu & $31,06 \mathrm{~g}$ & $\pm 30,90$ \\
D. auratus & $6,22 \mathrm{~g}$ & $\pm 3,66$ \\
E. argenteus & $8,34 \mathrm{~g}$ & $\pm 4,03$ \\
\hline
\end{tabular}


Os espécimes de L. alexandrei e L. jocu apresentam os mesmos hábitos alimentares, inclusive já foi verificada a formação de cardumes compostos pela associação dessas duas espécies, provavelmente utilizando-se do comportamento de agregação para proteção nos estágios de vida iniciais (Osório; Godinho \& Lotufo, 2011). No entanto, neste estudo os indivíduos de L. alexandrei apresentaram-se menores e mais pesados que os indivíduos de $L$. jocu, indicando que esta espécie exibe melhores condições nutritivas no que diz respeito à exploração do ambiente de manguezal, porém, os resultados obtidos ao final das coletas não foram suficientes para expressar a relação entre esses organismos de maneira segura.

Por meio da Anova foi observada diferença significativa apenas para a espécie L. jocu $\left(\mathrm{F}_{5,56}=3,8327, \mathrm{p}=0,0468\right)$, considerando os meses de amostragem e o peso de cada espécie dominante. As demais espécies: L. alexandrei $\left(\mathrm{F}_{5,57}=1,5977, \mathrm{p}=0,17547\right) ;$ D. auratus $\left(\mathrm{F}_{4,18}=\right.$ $2,8330, \mathrm{p}=0,05525)$ e E. argenteus: $\left(\mathrm{F}_{4,61}=0,27402, \mathrm{p}=0,89364\right)$ não apresentaram diferença estatística (Figura 7).

Em trabalhos de caracterização realizados em áreas de estuário e manguezal por Santos et al. (2002), Carvalho-Neta e Castro (2008) e Silva-Junior et al. (2013), com base na análise macroscópica das gônadas, comprimento e peso dos organismos amostrados, constatou-se que a maior parte da ictiofauna nesses ambientes é composta por indivíduos jovens, corroborando com os dados listados neste estudo.

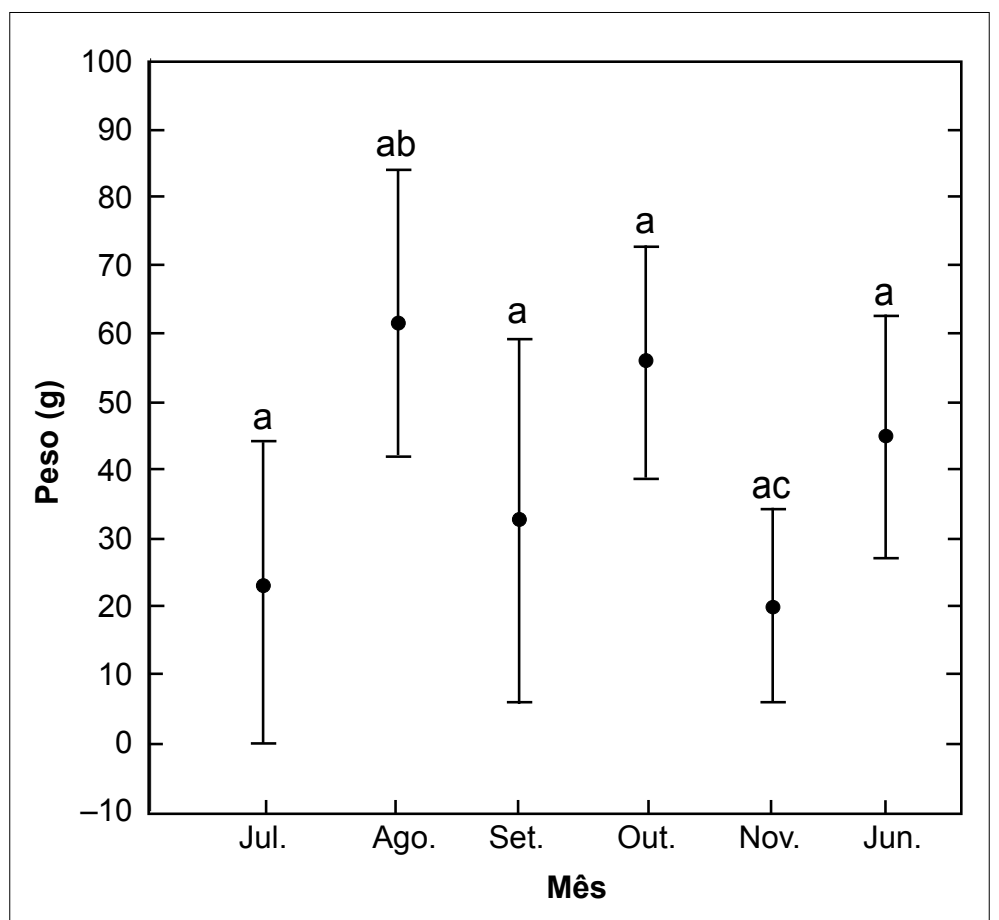

Figura 7 - Peso \pm erro padrão (g) de L. jocu ao longo dos meses amostrais (junho a novembro) no manguezal da Praia de Arpoeiras, no município de Acaraú - CE Nota: letras diferentes indicam diferenças significativas de acordo com o teste Tukey de comparações múltiplas.

A análise dos estágios de maturação gonadal de todos os organismos analisados indicou que os espécimes estavam imaturos, impossibilitando a sexagem e a definição do comprimento da primeira maturação sexual da ictiofauna associada à referida área de manguezal. Com base no período de amostragem, não foi possível determinar épocas de 
desova. No entanto, estudos anteriores revelaram que peixes do gênero Lutjanus, L. jocu e L. alexandrei são endêmicas e dependentes de áreas costeiras, como manguezais e recifes, principalmente durante seu período reprodutivo (Osório; Godinho \& Lotufo, 2011; Pinheiro \& Farias, 2016; Aschenbrenner; Hackradt \& Ferreira, 2016), assim como os membros da família Gerreidae coletados nesse estudo, D. Auratus e E. Argenteus (Viana et al., 2016; Pereira \& Pessanha, 2017; Silva et al., 2018). A ausência de organismos adultos na área amostrada possivelmente indica que os indivíduos coletados não completam seu ciclo de vida no mangue (Pinheiro \& Goitein, 2014), utilizando-o apenas como berçário e zona de alimentação.

\section{CONCLUSÃO}

A tarrafa é o método de captura mais eficiente para amostrar a ictiofauna em bosques de mangue quando comparada aos anzóis e manzuás.

O manguezal da Praia de Arpoeiras é dominado por quatro espécies de peixes endêmicos: Lutjanus alexandrei, Lutjanus jocu, Diapterus auratus e Eucinostomus argenteus. A icitiofauna é composta basicamente por espécimes jovens.

A maioria das espécies amostradas é de importância econômica para a pesca, o que justificaria a realização de estudos com escala temporal mais ampla, de modo a elucidar a dinâmica populacional e as estratégias reprodutivas para esses organismos em áreas de manguezal.

\section{REFERÊNCIAS BIBLIOGRÁFICAS}

Alves, R.R.N. \& Nishida, A.K. A ecdise do caranguejo-uçá, Ucides cordatus L. (Decapoda, Brachyura) na visão dos caranguejeiros. Interciência, Caracas, v. 27, n. 3, 2001. Disponível em: <https:/ / bit.ly/2ykn4Sh>. Acesso em: 18 fev. 2018.

Araújo, M.E.; Teixeira, J.M. \& Oliveira, A.M.E. Ictiofauna marinha do estado do Ceará, Brasil: III. Actinopterygii de estuários. Arquivos de Ciências do Mar, v. 33, n. 1-2, p. 139-142, 2000. Disponível em: <https:/ / bit.ly/2E9BNT4>. Acesso em: 7 fev. 2019.

Araújo, M.E.; Teixeira, J.M. \& Oliveira, A.M.E. Peixes estuarinos marinhos do Nordeste Brasileiro. Edições UFC, Fortaleza, 2004.

Araújo, M.V. et al. Análise dos impactos ambientais causados em estuários: estudo do estuário do Rio Acaraú, Ceará-Brasil. Caminhos de Geografia, Uberlândia, v. 8, n. 24, p. 111123, 2007. Disponível em: <http:/ / twixar.me/Cn2K>. Acesso em: 2 maio 2019.

Aschenbrenner, A.; Hackradt, C.W. \& Ferreira, B.P. Spatial variation in density and size structure indicate habitat selection throughout life stages of two Southwestern Atlantic snappers. Marine environmental research, v. 113, p. 49-55, 2016. Disponível em: < http:// twixar.me/Xq3n>. Acesso em: 4 maio 2019.

Barletta, M. et al. The role of salinity in structuring the fish assemblages in a tropical estuary. Journal of fish biology, Hoboken, v. 66, n. 1, 2005. Disponível em: <https://bit. ly/2CJlRrv>. Acesso em: 18 jan. 2018.

Basilio, T.H. et al. Ictiofauna do estuário do rio Curu, Ceará, Brasil. Arquivos de Ciências do Mar, v. 42, n. 2, p. 81-88, 2009. Disponível em: <https://bit.ly/2RS7Cog>. Acesso em: 5 fev. 2019. 
Benzeev, R., Hutchinson, N. \& Friess, D.A. Quantifying fisheries ecosystem services of mangroves and tropical artificial urban shorelines. Hydrobiologia, v. 803, p. 225-237, 2017. Disponível em: <https:/ / bit.ly/2thn6HA>. Acesso em: 12 fev. 2019.

Brehmer, P. et al. Does coastal lagoon habitat quality affect fish growth rate and their recruitment? Insights from fishing and acoustic surveys. Estuarine, Coastal and Shelf Science, Rio de Janeiro, v. 126, 2013. Disponível em: <https://bit.ly/2yDvWl1>. Acesso em: 13 mar. 2018.

Carvalho-Neta, R.N.F. \& Castro, A.C.L. Diversidade das assembleias de peixes estuarinos da Ilha dos Caranguejos, Maranhão. Arquivos de Ciências do Mar, v. 41, n. 1, p. 48-57, 2008. Disponível em: <https:/ / bit.ly/2SP5xwO>. Acesso em: 9 fev. 2019.

Catella, A.C. Aspectos ecológicos e culturais da pescaria de anzol. Embrapa Pantanal-Artigo de divulgação na mídia (INFOTECA-E), Corumbá, n. 108, p. 1-4, 2007. Disponível em: <https://bit.ly/2KibpqY>. Acesso em: 6 fev. 2019.

Cattani, A.P. et al. Avaliação de dispositivos para redução da ictiofauna acompanhante na pesca de arrasto dirigida ao camarão sete-barbas. Boletim do Instituto de Pesca, v. 38, n. 4, p. 333-348, 2012. Disponível em: <https:/ / bit.ly/2RXaL5a>. Acesso em: 7 fev. 2019.

Chaves, P.T. \& Vendel, A.L. Análise comparativa da alimentação de peixes (Teleostei) entre ambientes de marisma e de manguezal num estuário do sul do Brasil (Baía de Guaratuba, Paraná). Revista Brasileira de Zoologia, Curitiba, v. 25, n. 1, 2008. Disponível em: <https:/ / bit.ly/2CKVykK>. Acesso em: 22 fev. 2018.

Chaves, P.T.C. \& Serenato, A. Diversidade de dietas na assembleia de linguados (Teleostei, Pleuronectiformes) do manguezal da Baía de Guaratuba, Paraná, Brasil. Revista Brasileira de Oceanografia, São Paulo, v. 46, n. 1, 1998. Disponível em: <https://bit.ly/2OsXSTu>. Acesso em: 24 mar. 2018.

Duarte, C.; Deus, C.P. \& Py-Daniel, L.R. Comparação da eficiência de dois apetrechos de coleta de peixes em praias no baixo rio Purus, Amazonas, Brasil. Acta Amazonica, v. 43, n. 3, 2012. Disponível em: <https:/ / bit.ly/2GEqt37>. Acesso em: 4 fev. 2019.

Duke, N. C. Mangrove Floristics and Biogeography Revisited: Further Deductions. FISHBASE, 2017. Disponível em: < http://twixar.me/W8HK>. Acesso em: 26 nov. 2017.

Fonseca, A.F. \& Souza, R.A.L. Caracterização ecológica de algumas espécies da fauna acompanhante do camarão capturado com puçá de arrasto na zona estuarina do Rio Taperaçu. Tropical Journal of Fisheries and Aquatic Sciences, Bragança-PA, v. 6, n. 1, p. 33-47, 2013. Disponível em: <https://bit.ly/2TRE9M8>. Acesso em: 7 fev. 2019.

Fonteles-Filho, A.A. Oceanografia, biologia e dinâmica populacional de recursos pesqueiros. Universidade Federal do Ceará, 236 p., Fortaleza, 2011.

Funceme. Calendário das chuvas no estado do Ceará. Ceará, 2017. Disponível em: $<$ https:/ / bit.ly/2pVr3A8>. Acesso em: 19 fev. 2018.

Giglio, V.J. \& Freitas, M.O. Caracterização da pesca artesanal com rede de camboa na Reserva Extrativista do Cassurubá, Bahia. Biotemas, Florianópolis, v. 26, n. 2, 2013. Disponível em: <https:/ / bit.ly/2AbD7CZ>. Acesso em: 12 fev. 2018.

Gomes, C. et al. The role of drifting objects in pelagic fisheries in the Southeastern Caribbean. Fisheries Research, v. 34, n. 1, p. 47-58, 1998. Disponível em: < http://twixar.me/ d7qK>. Acesso em: 2 maio 2019. 
Grande, H. et al. Diversity of settlement-stage reef fishes captured by light-trap in a tropical south-west Atlantic Ocean coastal reef system. Journal of fish biology, 2018. Disponível em: <https://bit.ly/2tjHFTw>. Acesso em: 8 fev. 2019.

Hamley, J.1.M. Review of gillnet selectivity. Journal Fish Research Board Canadá, Toronto, v. 32, n. 11, 1975. Disponível em: <https:// bit.ly/2MX9h9W>. Acesso em: 26 fev. 2018.

IBGE. Censo Demográfico 2010 - Características Gerais da População. Resultados da Amostra. IBGE, 2010. Disponível em: <https:// bit.ly/2DmtAul>. Acesso em: 15 jan. 2019.

Ignácio, J.M. \& Spach, H.L. Variação entre o dia e a noite nas características da ictiofauna do infralitoral raso do Maciel, Baía de Paranaguá, Paraná. Revista Brasileira de Zoociências, v. 11, n. 1, p. 25-37, 2009. Disponível em: <https://bit.ly/2thsElo>. Acesso em: 7 fev. 2019.

Lee, S.Y. et al. Ecological role and services of tropical mangrove ecosystems: a reassessment. Global Ecology and Biogeography, Australian, v. 23, p. 726-743, 2014. Disponível em: <https:/ / bit.ly/2SuaagD>. Acesso em: 4 fev. 2019.

Lessa, R. \& Nóbrega, M.F. Peixes marinhos do Nordeste do Brasil_-Programa REVIZEE_SCORE/NE. Recife, 2009. Disponível em: < http://twixar.me/f8HK>. Acesso em: 21 jan. 2018.

Lira, A.K.F.\&Teixeira,S.F.Ictiofauna da praia deJaguaribe, Itamaracá,Pernambuco.Iheringia, Sér. Zool, v. 98, n. 4, p. 475-480, 2008. Disponível em: <https://bit.ly/2GEo3S8>. Acesso em: 7 fev. 2019.

Maia, R.C. Manguezais do Ceará. Imprima, 55 p., Recife, 2016.

Manson, F.J. et al. An evaluation of the evidence for linkages between mangroves and fisheries: a synthesis of the literature and identification of research directions. Oceanography and marine biology, Londres, v. 43, 2005. Disponível em: <https://bit.ly/2In9cJ8>. Acesso em: 20 jan. 2018.

Masih-Neto et al. Biodiversidade da ictiofauna nos currais de pesca no litoral de Acaraú, Ceará, Brasil. Arquivos de Ciências do Mar, Fortaleza, v. 50, n. 2, 2017. Disponível em: <https:/ / bit.ly/2OsYI2A>. Acesso em: 27 maio 2018.

Mattos, P.P. et al. Etnoconhecimento e percepção dos povos pesqueiros da Reserva Ponta do Tubarão acerca do ecossistema manguezal. Revista Brasileira de Biociências, v. 10, n. 4, p. 481, 2012. Disponível em: <https:/ / bit.ly/2N58eVL>. Acesso em: 4 fev. 2019.

Melo, F.A.G. et al. Guia de identificação dos peixes do estuário dos rios Timonha e Ubatuba. Sieart, Parnaíba, 2015.

Menezes, N.A. et al. Taxonomic review of the species of Mugil (Teleostei: Perciformes: Mugilidae) from the Atlantic South Caribbean and South America, with integration of morphological, cytogenetic and molecular data. Zootaxa, v. 3941, n. 4, p. 600-600, 2015. Disponível em: < http://twixar.me/2jHK>. Acesso em: 18 jan. 2018.

Moraes, L.E. et al. Ictiofauna demersal da plataforma continental interna ao largo de Ilhéus, Bahia, Brasil. Biota Neotropica, v. 9, n. 4, p. 163, 2009. Disponível em: <https://bit. ly/2SMawym>. Acesso em: 4 fev. 2019.

Moraes, R.L.G.D. Pesca, parasitismo e dieta alimentar da baúna Lutjanus alexandrei Moura \& Lindeman, 2007, nos ambientes costeiros do litoral sul de Pernambuco. Dissertação de mestrado, Programa de Pós-Graduação em Oceanografia, Universidade Federal de Pernambuco. 1 p., Recife, 2012. Disponível em: <https:/ / bit.ly/2DmwBv1>. Acesso em: 18 jan. 2019. 
Moura, R.L. \& Lindeman, K.C. A new species of snapper (Perciformes: Lutjanidae) from Brazil, with comments on the distribution of Lutjanus griseus and L. apodus. Zootaxa, v. 1422, n. 3, p. 31-43, 2007. Disponível em: <https://216.92.145.68/zootaxa/2007f/ zt01422p043.pdf>. Acesso em: 13 jan. 2018.

Neves, L.M. et al. Uso do manguezal de Guaratiba, Baía de Sepetiba, Rio de Janeiro, pelo peixe-rei Atherinella brasiliensis (Quoy \& Gaimard) (Atheriniformes, Atherinopsidae). Revista Brasileira de Zoologia, Rio de Janeiro, v. 23, n. 2, 2006. Disponível em: <https:/ / bit.ly/2MnSDPE>. Acesso em: 24 jan. 2018.

Oliveira-Silva, J.T.; Peso-Aguiar, M.C. \& Lopes, P.R.D. Ictiofauna das praias de Cabuçu e Berlinque: uma contribuição ao conhecimento das comunidades de peixes na Baía de Todos os Santos-Bahia-Brasil. Biotemas, v. 21, n. 4, p. 105-115, 2008. Disponível em: <https:// bit. ly/2SJLW16>. Acesso em: 8 fev. 2019.

Osório, F.M.; Godinho, W.O. \& Lotufo, T.M.C. Ictiofauna associada às raízes de mangue do estuário do rio Pacoti-CE, Brasil. Biota Neotropica, Campinas, v. 11, n. 1, 2011. Disponível em: <https:/ / bit.ly/2QOSCGs>. Acesso em: 21 fev. 2018.

Paiva, A.C.G.; Chaves, P.T.C. \& Araújo, M.E. Estrutura e organização trófica da ictiofauna de águas rasas em um estuário tropical. Revista Brasileira de Zoologia, v. 25, n. 4, p. 647-661, 2008. Disponível em: <https://bit.ly/2Gqjk74>. Acesso em: 5 fev. 2019.

Pereira, J.A. \& Pessanha, A.L.M. Ecologia trófica de Diapterus auratus Ranzani, 1840 (perciformes: gerreidae), em um estuário tropical semiárido do Nordeste brasileiro, in Anais do II Congresso Internacional da Diversidade do Semiárido, Paraíba, 2017. Disponível em: <https:/ / bit.ly/2Do3KpY>. Acesso em: 15 jan. 2019.

Pichler, H.A. et al. Influência do artefato de pesca na caracterização da ictiofauna em planícies de maré. Cadernos da Escola de Saúde, v. 1, n. 1, 2017. Disponível em: < https:/ / bit. ly/2Suitsw>. Acesso em: 4 fev. 2019.

Pinheiro, M.S.S. \& Goitein, R. Estrutura de uma população e aspectos biológicos de Mugil curema valenciennes, 1836 (Pisces, Mugilidae), em um manguezal da Raposa, Brasil. Revista de Ciências da Saúde, São Luiz, v. 16, n. 2, 2014. Disponível em: <https:/ / bit.ly/2KnCXOM>. Acesso em: 27 jan. 2018.

Pinheiro, W.M. \& Farias, A.C.S. Composição específica, bioecologia e ecomorfologia da ictiofauna marinha oriunda da pesca de pequena escala. Bol. Inst. Pesca, São Paulo, v. 42, n. 1, p. 181-194, 2016. Disponível em: <https:/ / bit.ly/2E8mJoI>. Acesso em: 9 fev. 2019.

Portella, G.G.D. \& Medeiros, R.P. Modificações estruturais nas redes de arrasto de camarão: percepção dos pescadores e implicações para a gestão em uma área marinha protegida. Bol. Inst. Pesca, São Paulo, v. 42, n. 1, p. 1-16, 2016. Disponível em: <https://bit.ly/2WTuBls>. Acesso em: 8 fev. 2019.

Possamai, B.; Rosa, L. \& Corrêa, M.F.M. Seletividade de armadilhas e atrativos na captura de pequenos peixes e crustáceos em ambientes estuarinos. Brazilian Journal of Aquatic Science and Technology, v. 18, n. 2, p. 11-17, 2014. Disponível em: <https://bit.ly/2N8xSck>. Acesso em: 7 fev. 2019.

Rezende, C.E. et al. An economic valuation of mangrove restoration in Brazil. Ecological Economics, v. 120, p. 296-302, 2015. Disponível em: <https://bit.ly/2SPXFeC>. Acesso em: 3 fev. 2019. 
Ribeiro, O.M. \& Zuanon, J. Comparação da eficiência de dois métodos de coleta de peixes em igarapés de terra firme da Amazônia Central. Acta Amazonica, v. 36, n. 3, p. 389-394, 2006. Disponível em: <https://bit.ly/2RGMDsc>. Acesso em: 18 jan. 2019.

Rocha, S.S. Diferença entre dois métodos de coleta utilizados na captura de crustáceos decápodes em um rio da Estação Ecológica Juréia-Itatins, São Paulo. Iheringia, Sér. Zool, v. 100, n. 2, p. 116-122, 2010. Disponível em: <https:/ / bit.ly/2SH6Td9>. Acesso em: 6 fev. 2019.

Sanches, E.G. \& Sebastiani, E.F. Atratores e tempo de submersão na pesca artesanal com armadilhas. Biotemas, v. 22, n. 4, p. 199-206, 2009. Disponível em: <https:/ / bit.ly/2SyFJ90>. Acesso em: 11 fev. 2019.

Santos, C. et al. A ictiofauna em duas planícies de maré do setor euhalino da Baía de Paranaguá, PR. Instituto de Pesca, São Paulo, v. 28, n. 1, p. 40-60, 2002. Disponível em: <https:/ / bit.ly/2Id4hzo>. Acesso em: 7 fev. 2019.

Santos, N.M. \& Lana, P. Present and past uses of mangrove wood in the subtropical Bay of Paranaguá (Paraná, Brazil). Ocean \& Coastal Management, Amsterdam, v. 148, 2017. Disponível em: <https://bit.ly/2tZE90D>. Acesso em: 21 mar. 2018.

Saraiva, S.Z.R. Caracterização socioeconômica da pesca artesanal na Ilha dos Coqueiros em Acaraú, Estado do Ceará. Fortaleza, 2015. 63 f. Dissertação de mestrado, Ciências Marinhas Tropicais, Instituto de Ciências do Mar, Universidade Federal do Ceará, Fortaleza, 2015. Disponível em: <http:/ / twixar.me/kh2K>. Acesso em: 2 maio 2019.

Sheridan, P. \& Hays, C. Are mangroves nursery habitat for transient fishes and decapods? Wetlands, Madison, v. 23, n. 2, 2003. Disponível em: <https:// bit.ly/2tyJnAp>. Acesso em: 25 fev. 2018.

Silva, V.E. et al. Spatial distribution of juvenile fish species in nursery grounds of a tropical coastal area of the south-western Atlantic. Acta Ichthyologica et Piscatoria, v. 48, n. 1, 2018. Disponível em: < http:/ / twixar.me/J1Kn>. Acesso em: 4 maio 2019.

Silva-Júnior, M.G. et al. Caracterização da ictiofauna em três canais de maré do estuário do rio Paciência, ilha de São Luís, estado do Maranhão. Arquivos de Ciências do Mar, v. 46, n. 1, p. 5-21, 2013. Disponível em: <https:/ / bit.ly/2GGEqxn>. Acesso em: 5 fev. 2019.

Souza, C.A. et al. Biodiversidade e conservação dos manguezais: importância bioecológica e econômica, p. 16-56, in Pinheiro, M.A.A. \& Talamoni, A.C.B. (Org.). Educação Ambiental sobre Manguezais. Unesp, 165 p., São Vicente, 2018. Disponível em: < http://twixar.me/ PmqK>. Acesso em: 11 mar. 2018.

Szpilman, M. Peixes marinhos do Brasil: guia prático de identificação. MAUAD Editora Ltda., Rio de Janeiro, 2000.

Teixeira, T.P. et al. Diversidade das assembleias de peixes nas quatro unidades geográficas do rio Paraíba do Sul. Iheringia. Série Zoologia, Porto Alegre, v. 95, n. 4, 2005. Disponível em: <https:/ / bit.ly/2OpgLql>. Acesso em: 8 maio 2018.

Thiers, P.R.L; Meireles, A.J.A. \& Santos, J.O. Manguezais na costa oeste cearense - preservação permeada de meias verdades. Editora Imprensa Universitária, Fortaleza, p. 126, 2016.

Tomlinson, P.B. The botany of mangroves. 2. ed., 418 p., University Press Cambridge, 2016.

Torres, C.M.et al. Caracterização da pesca de tainhas no município de porto de Pedras. Revista Brasileira de Engenharia de Pesca, v. 2, n. 3, p. 6-17, 2009. Disponível em: <https://bit. ly/2TOi2pw>. Acesso em: 8 fev. 2019. 
Tran, L.X. \& Fischer, A. Spatiotemporal changes and fragmentation of mangroves and its effects on fish diversity in Ca Mau Province (Vietnam). Journal of Coastal Conservation, v. 21, p. 355-368, 2017. Disponível em: <https:// bit.ly/2GGp8c2>. Acesso em: 2 fev. 2019.

Vazzoler, A.E.A.M. Biologia da reprodução de peixes teleósteos: teoria e prática. Eduem, 191 p., São Paulo, 1996.

Viana, A.P. et al. Length-weight relations of 70 fish species (actinopterygii) from tropical coastal region of Pernambuco, northeast Brazil. Acta Ichthyologica et Piscatoria, v. 46, n. 3, 2016. Disponível em: <http://twixar.me/T3Kn>. Acesso em: 4 maio 2019.

Xavier, J.H.A. et al. Fish assemblage of the Mamanguape Environmental Protection Area, NE Brazil: abundance, composition and microhabitat availability along the mangrove-reef gradient. Neotropical Ichthyology, v. 10, n. 1, p. 109-122, 2012. Disponível em: <https:// bit. ly/2USNWkY>. Acesso em: 6 fev. 2019. 